\title{
Unique properties and behavior of nonmercerized type- \\ II cellulose nanocrystals as carbon nanotube biocompatible dispersants
}

Jose M González-Domínguez, ${ }^{1 *}$ Alejandro Ansón-Casaos, ${ }^{1}$ Laura Grasa, ${ }^{2,3,4}$ Luis Abenia, ${ }^{1 \ddagger}$ Alba Salvador, ${ }^{1 \ddagger}$ Eduardo Colom, ${ }^{1}$ Jose E. Mesonero, ${ }^{2,3,4}$ J. Enrique GarcíaBordejé, ${ }^{1}$ Ana M. Benito, ${ }^{1}$ Wolfgang K. Maser. ${ }^{1}$

AUTHOR ADDRESSES: 1) Group of Carbon Nanostructures and Nanotechnology, Instituto de Carboquímica ICB-CSIC. C/ Miguel Luesma Castán 4, 50018 Zaragoza (Spain); 2) Departamento de Farmacología y Fisiología, Facultad de Veterinaria, Universidad de Zaragoza, C/ Miguel Servet s/n, 50013, Zaragoza (Spain); 3) Instituto de Investigación Sanitaria de Aragón (IIS Aragón); 4) Instituto Agroalimentario de Aragón (IA2).

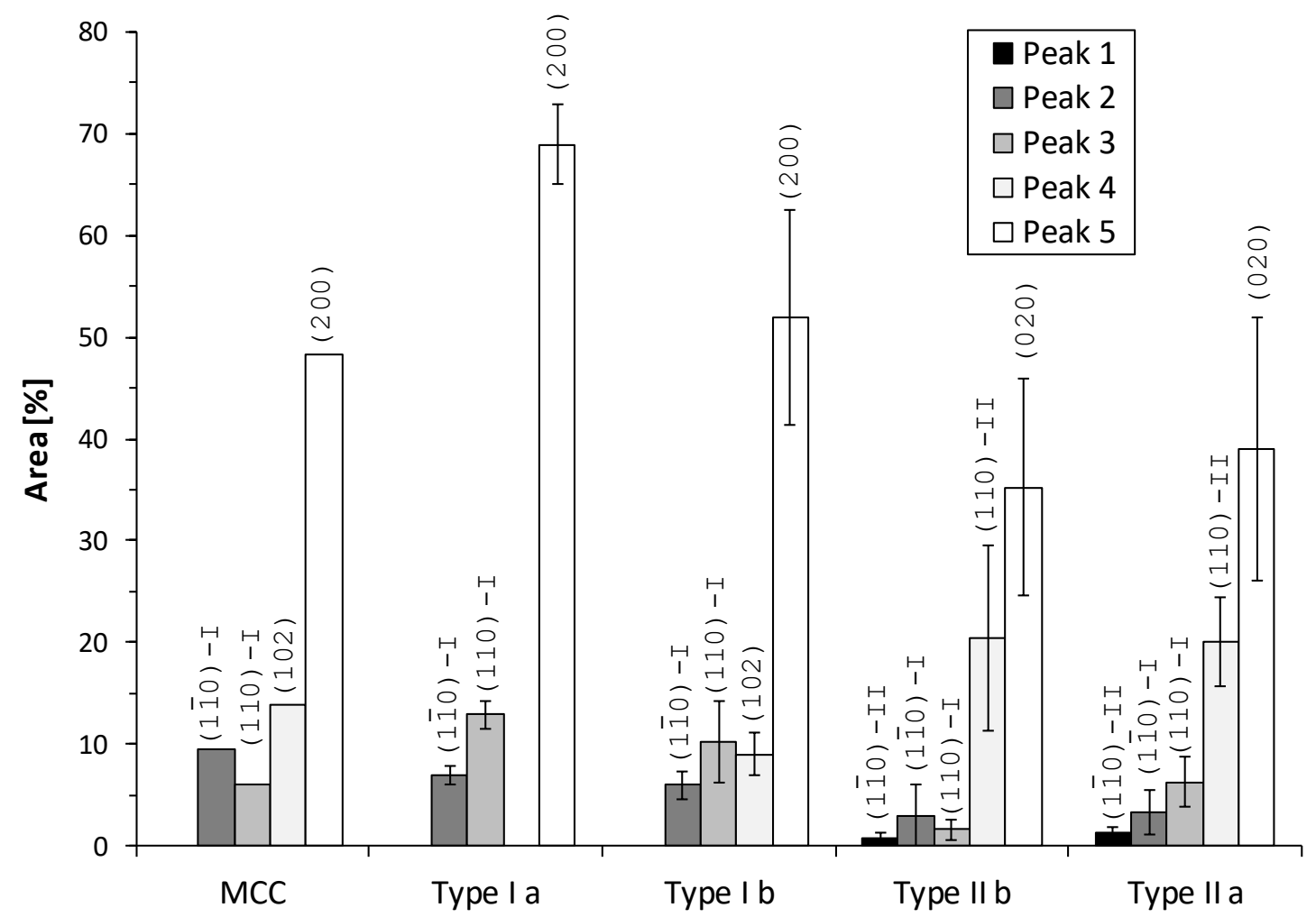

Figure S1.- Summary of peak areas under the main components from deconvoluted X-Ray diffractograms (Figure 1 and Table 1). Total areas do not add up 100\% since only the most representative features for each NCC allomorph have been considered.

The largest contribution to the XRD profile is given by peak 5, namely (200) in type-I or (020) in type-II based samples. As MCC turns into type-I NCC, this component raises significantly ( 20\%), pointing to an actual increase in the allomorphic purity of the sample. As a matter of fact, the specific type-I components of MCC add up a 78\% of the total area, while for pure type-I NCC it is $88 \%$. As the experimental conditions shift to those leading to type-II NCC samples and mixtures, the peak 5 component steadily decreases in specific weight, until reaching a stabilization at $35-40 \%$ for the NCC samples with dominant type-II character. A similar trend is 
observed on peak 3, the (110) diffraction of type-I allomorph, but the peak 2 component only shows a progressive decrease from MCC to type-I and type-II samples. When staring at type-II based samples, peaks 1 and 4 acquire a noticeable relevance, becoming the most representative together with peak 5. However, there are still some type-I features (peaks 2 and 3 ) regardless of the experimental conditions employed, meaning that type-l allomorph is very difficult to be fully converted into type-II. According to the relative ratio between characteristic type-I and type-II peak areas in Figure 2, a percentage of each allomorph type in NCC samples can be obtained. ${ }^{1}$

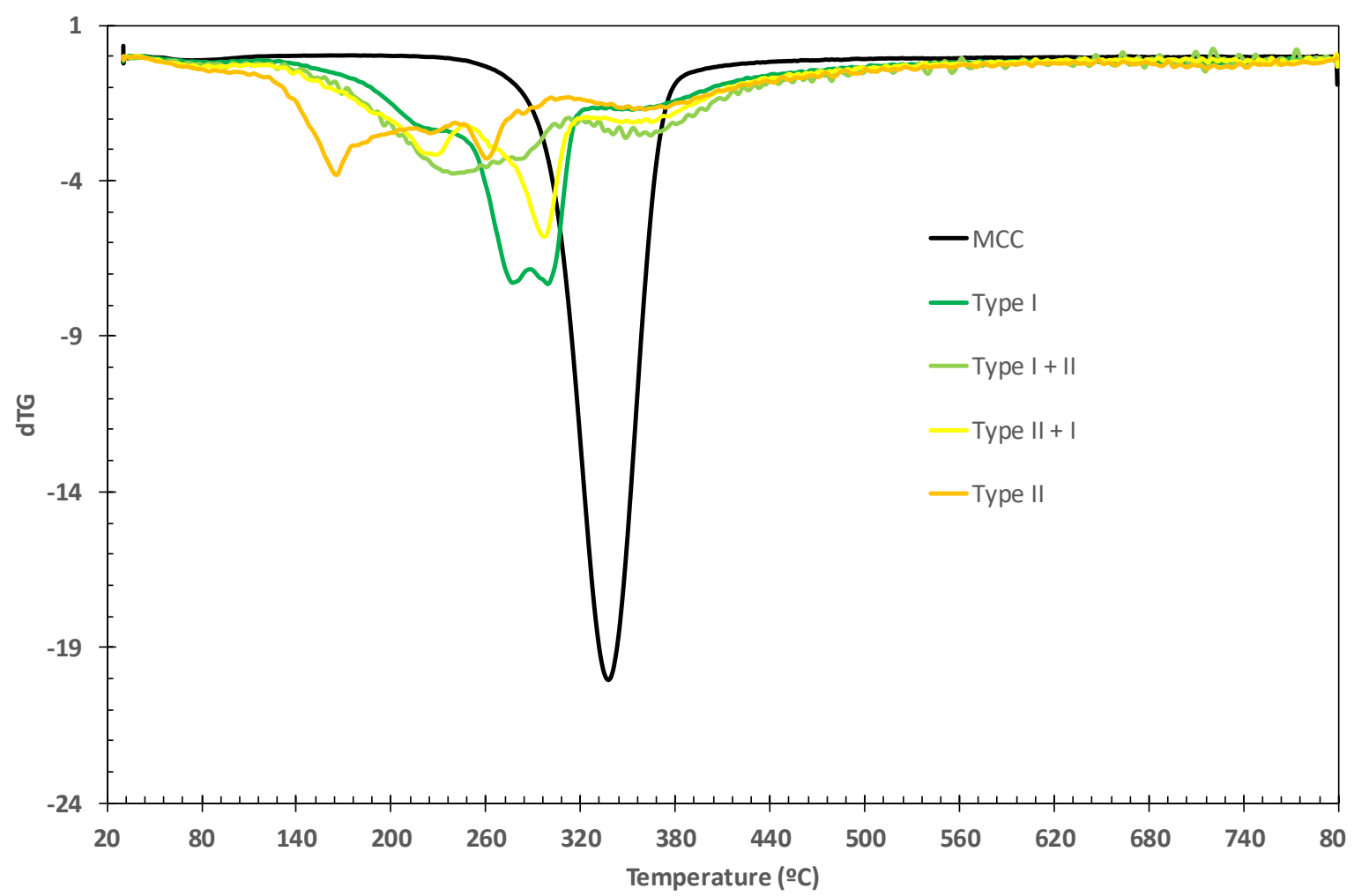

Figure S2.- Differential thermograms (dTG) obtained from the TGA in inert atmosphere.

\footnotetext{
${ }^{1}$ Merlini, A.; Souza, V. C. D. E.; Gomes, R. M.; Coirolo, A.; Machado, R. A. F.; Merlini, A. Effects of reaction conditions on the shape and crystalline structure of cellulose nanocrystals. Cellulose Chem. Technol. 2018, $52,325-335$.
} 
Table S1. Oxygen index data calculated from TGAs in inert atmosphere.

\begin{tabular}{|c|c|c|c|}
\hline $\begin{array}{c}\text { NCC } \\
\text { sample }\end{array}$ & $\begin{array}{c}\text { TGA Residue } \\
(w t \%)\end{array}$ & $\%$ OI & Flammable? \\
\hline Type-I & 19.6 & 25.3 & YES \\
\hline Type-I + II & 21.6 & 26.1 & In the limit \\
\hline Type-II + I & 22.6 & 26.6 & NO \\
\hline Type II & 25.2 & 27.6 & NO \\
\hline
\end{tabular}

Flame resistance was evaluated throughout the empirical equation of Van Krevelen $(\% \mathrm{OI}=17.5$ $+0.4 \cdot \mathrm{R}) .{ }^{2}$ Where $\% \mathrm{OI}$ is the oxygen index and $\mathrm{R}$ stands for the solid residue in wt\%. The limiting $\mathrm{OI}$, below which a material can be considered flammable is $26 \%$, so the type-II allomorphs produced in this work have a clear resistance to flame Sulfate species are well-known flame retardant additives to polymers. ${ }^{3,4}$ So, results from Table S1 could be attributed to a higher sulfate content of type-II made by acid hydrolysis (see results and discussion), which can arguably come from the longer exposure time of $\mathrm{MCC}$ to the $\mathrm{H}_{2} \mathrm{SO}_{4}$ in our experimental procedure, and also the fact that type-II allomorphs have a larger number of accessible-OHs for esterification (about double) than type-I as per their crystalline structure. ${ }^{5}$ This unique difference between types I and II of NCC has not yet been highlighted, since commonly mercerized type-II NCC does not rely in $\mathrm{H}_{2} \mathrm{SO}_{4}$ to break the hydrogen bonding structure of native type-I, hence the eventual sulfation degree should not differ much from one allomorph to another. Our findings suggest that type-II NCC produced by acid hydrolysis could be envisaged as nanocomposite fillers with flame retardant capability. The limiting OI found here for non-mercerized type-II based NCC samples surpass that of commercial polymer-based foams ( $\mathrm{OI}=22-25 \%)$, and these values were achieved without foaming or compositing NCC, which could reach OI values as high as $34 .{ }^{6}$

${ }^{2}$ González-Domínguez, J. M.; Ansón-Casaos, A.; Castell, P.; Díez-Pascual, A. M.; Naffakh, M.; Ellis, G.; Gómez, M. A.; Teresa Martínez, M. Integration of Block Copolymer-Wrapped Single-Wall Carbon Nanotubes into a Trifunctional Epoxy Resin. Influence on Thermal Performance. Polym. Degrad. Stab. 2010, 95 (10), 2065-2075.

${ }^{3}$ Lu, H.; Hu, Y.; Yang, L.; Wang, Z.; Chen, Z.; Fan, W. Study of the Fire Performance of Magnesium Hydroxide Sulfate Hydrate Whisker Flame Retardant Polyethylene. Macromol. Mater. Eng. 2004, 289 (11), 984-989.

${ }^{4}$ Murariu, M.; Bonnaud, L.; Yoann, P.; Fontaine, G.; Bourbigot, S.; Dubois, P. New Trends in Polylactide (PLA)-Based Materials: "Green" PLA-Calcium Sulfate (Nano)Composites Tailored with Flame Retardant Properties. Polym. Degrad. Stab. 2010, 95 (3), 374-381.

${ }^{5}$ Flauzino Neto, W. P.; Putaux, J. L.; Mariano, M.; Ogawa, Y.; Otaguro, H.; Pasquini, D.; Dufresne, A. Comprehensive Morphological and Structural Investigation of Cellulose I and II Nanocrystals Prepared by Sulphuric Acid Hydrolysis. RSC Adv. 2016, 6, 76017-76027.

${ }^{6}$ Lavoine, N.; Bergström, L. Nanocellulose-Based Foams and Aerogels: Processing, Properties, and Applications. J. Mater. Chem. A 2017, 5 (31), 16105-16117. 

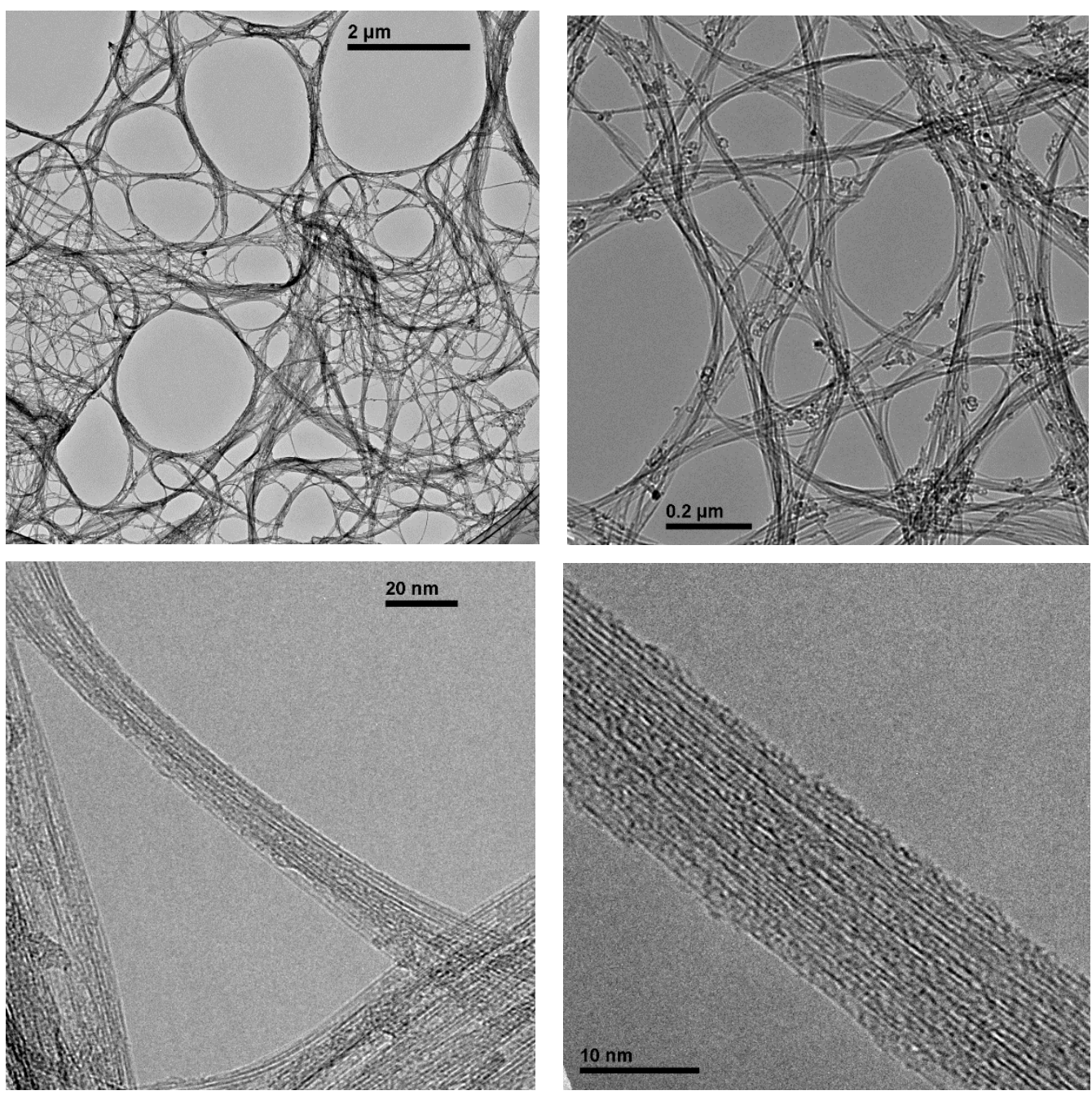

Figure S3.- TEM images of the initial SWCNTs (AP Carbon Solutions, treated with air $+\mathrm{HCl}$ reflux). Only for the TEM purpose, this sample was dispersed and centrifuged in SDBS surfactant in order to be well deposited onto the grid. 